\title{
Synergy of gatifloxacin with cefoperazone and cefoperazone-sulbactam against resistant strains of Pseudomonas aeruginosa
}

\author{
Correspondence \\ B. Meenashi Vanathi \\ meenashivanathi@gmail.com
}

Received 1 March 2008

Accepted 20 August 2008

\author{
N. Sivagurunathan, ${ }^{1,2}$ S. Krishnan, ${ }^{2}$ J. Venkat Rao, ${ }^{1}$ \\ Anantha Naik Nagappa, ${ }^{1}$ V. M. Subrahmanyam ${ }^{1}$ and B. Meenashi Vanathi ${ }^{3}$ \\ ${ }^{1}$ Department of Pharmaceutical Biotechnology, Manipal College of Pharmaceutical Sciences, \\ Manipal University, Manipal, India \\ ${ }^{2}$ Department of Pharmaceutical Biotechnology, College of Pharmacy, Sri Ramakrishna Institute of \\ Paramedical Sciences, Coimbatore, India \\ ${ }^{3}$ Department of Pharmaceutical Biotechnology, Shree Devi College of Pharmacy, Airport Road, \\ Kenjar Village, Mangalore, Karnataka 574142, India
}

\section{INTRODUCTION}

Resistance to antimicrobial agents has resulted in morbidity and mortality from treatment failures, and increased health-care costs. Although defining the precise publichealth risk and estimating the increase in costs is not a simple undertaking, there is little doubt that the emergent antibiotic resistance is a serious global problem.

Despite improvements in antimicrobial therapy, Pseudomonas aeruginosa remains one of the most prominent Gramnegative bacteria causing nosocomial infections, and comprises $16 \%$ of nosocomial pneumonia cases, $12 \%$ of hospital-acquired urinary tract infections, $8 \%$ of surgical wound infections and $10 \%$ of bloodstream infections (Rossolini \& Mantengoli, 2005). This organism is uniquely problematic because of a combination of inherent resistance to many drug classes and its ability to acquire resistance to all relevant treatments (Rossolini \& Mantengoli, 2005). Resistance mechanisms include low outer-membrane permeability, multidrug efflux pumps (tetracycline, imipenem, fluoroquinolones and aminoglycosides) and the production of antibiotic-modifying enzymes such as metallo- $\beta$-lactamases (aminoglycosides and $\beta$-lactams) (Rossolini \& Mantengoli, 2005). The development of vaccines and new antimicrobial agents has not kept pace with resistance;

Abbreviations: CFP, cefoperazone; CFP-SUL, cefoperazone-sulbactam; FIC, fractional inhibitory concentration; GAT, gatifloxacin. therefore, the search for other methods of therapy, such as synergistic combinations, is necessary.

Combination therapy is used with the aim of expanding the antimicrobial spectrum, minimizing toxicity, preventing the emergence of resistant mutants during therapy and obtaining synergistic antimicrobial activity (Eliopoulos \& Moellering, 1991). Many antimicrobial combinations have been studied for synergy in vitro and in vivo against $P$. aeruginosa (Dawis et al., 2003; Fish et al., 2002; Gradelski et al., 2001; Mayer \& Nagy, 1999). In this study, we investigated the activity of gatifloxacin (GAT), alone and in combination with cefoperazone (CFP) and cefoperazone-sulbactam (CFP-SUL) (2:1), against 58 clinical isolates of $P$. aeruginosa.

\section{METHODS}

Bacterial isolates. A total of 58 non-replicate strains of $P$. aeruginosa, recently isolated from clinical specimens obtained from the Department of Microbiology, Sri Ramakrishna Hospital, Coimbatore, India, were included in the study. Overall, $62 \%$ of the isolates were obtained from the respiratory tract, $17 \%$ from urine and $21 \%$ from a variety of other sources. The isolates were identified by conventional methods and checked for purity by plating on MacConkey agar. P. aeruginosa ATCC 27853 was included as a quality-control strain.

Antimicrobial agents. Standard laboratory powders of GAT, CFP and SUL were used in this study. 
Antibiotic susceptibility testing. Antibiotic susceptibility testing was done on Mueller-Hinton ( $\mathrm{MH})$ agar using the Kirby-Bauer disc diffusion method with GAT, CFP and CFP-SUL. The results were interpreted according to the guidelines of the Clinical and Laboratory Standards Institute (formerly the National Committee for Clinical Laboratory Standards) (CLSI, 2000). The susceptible break points applied were: $\leqslant 2 \mu \mathrm{g} \mathrm{ml}^{-1}$ for GAT, $\leqslant 16 \mu \mathrm{g} \mathrm{ml}^{-1}$ for CFP and $\leqslant 16 \mu \mathrm{g} \mathrm{ml}^{-1}$ for CFP-SUL. Isolates of intermediate sensitivity were categorized as resistant, as the number of intermediate isolates was insignificant when compared with the whole sample. Accordingly, the isolates were categorized as: susceptible (to all agents tested), resistant (to each agent alone) and miscellaneous (resistant to more than one of the agents tested).

MIC determination. The MICs of each agent alone were determined by broth macrodilution using sterile glass test tubes containing $\mathrm{MH}$ broth (supplemented with magnesium and calcium cations). The inoculum contained $5 \times 10^{5}$ c.f.u. $\mathrm{ml}^{-1}$. The concentration ranges tested were: $0.125-16 \mu \mathrm{g}$ GAT ml ${ }^{-1}, 1-128 \mu \mathrm{g} \mathrm{CFP} \mathrm{ml}^{-1}$ and 1$128 \mu \mathrm{g}$ CFP-SUL $\mathrm{ml}^{-1}$. Antimicrobial solutions were prepared and freshly diluted on the day of testing. Each test was performed in duplicate (CLSI, 1999).

\section{Synergy testing}

Chequerboard method. Chequerboard synergy testing was performed in sterile glass test tubes using the macrodilution technique. The concentration ranges and inoculum were the same as used for MIC determination. Antimicrobial solutions were prepared and freshly diluted on the day of the test. Each test was performed in duplicate. Fractional inhibitory concentrations (FICs) were calculated as: (MIC of drug A or B in combination)/(MIC of drug $\mathrm{A}$ or $\mathrm{B}$ alone), and the FIC index was obtained by adding the FIC values. FIC values were interpreted as synergistic if values were $\leqslant 0.5$, additive or indifferent for values $>0.5$ to 4.0 and antagonistic for values >4.0 (Eliopoulos \& Moellering, 1991; Visalli et al., 1998).

Time-kill method. From each of the above-classified susceptibility groups, three strains (except for CFP-resistant strains, where two strains were used instead of three) were tested by a time-kill method as described by Bajaksouzian et al. (1996) and Visalli et al. (1998). Antimicrobial solutions were prepared and freshly diluted on the day of testing. All antimicrobial agents were tested alone and in combination. In each case, concentrations ranging from four times above to four times below the MIC were tested. Drug carryover was addressed as described by Bajaksouzian et al. (1996). Each test was performed in duplicate. Viability counts were performed in duplicate at $0,6,12$ and 24 h by plating on $\mathrm{MH}$ agar. Synergy was defined as a $\geqslant 2 \log _{10}$ decrease in the viable count with the combination at $24 \mathrm{~h}$ compared with that of the more active of each of the two compounds tested alone. Indifference was defined as $a \geqslant 2 \log _{10}$ increase or decrease in colony count at $24 \mathrm{~h}$ by the combination compared with that by the most active drug alone. Antagonism was defined as a $\geqslant 2$ $\log _{10}$ increase in colony count at $24 \mathrm{~h}$ by the combination compared with that by the most active drug alone (Bajaksouzian et al., 1996; Visalli et al., 1998).

Statistical determination. Statistical significance was determined using the McNemar test.

\section{RESULTS}

In susceptibility testing, $43 \%$ of the isolates were found to be resistant to GAT, $36 \%$ were resistant to CFP and $25 \%$ were resistant to CFP-SUL. The $\mathrm{MIC}_{50} / \mathrm{MIC}_{90}$ values of the agents are presented in Table 1 . The MIC ranges of GAT, CFP and CFP-SUL observed were 1-16, 8-128 and 8-64 $\mu \mathrm{g}$ $\mathrm{ml}^{-1}$, respectively. The chequerboard results are listed in Table 2. Synergistic FIC indices for the GAT + CFP combination were found in $21 / 58$ strains (36.2\%), which comprised 11/31 strains susceptible to all agents tested, 2/6 strains resistant to GAT alone, $2 / 2$ strains resistant to CFP alone and 6/19 strains classified as miscellaneous. For the GAT + CFP-SUL combination, synergistic FIC indices were found in $34 / 58$ strains (58.6\%), which included $17 / 31$ strains susceptible to all agents tested, 4/6 strains resistant to GAT alone, $2 / 2$ strains resistant to CFP alone and 11/19 strains classified as miscellaneous.

The results of the time-kill synergy tests are listed in Table 3. Time-kill studies revealed synergy in 6/11 strains $(54.5 \%)$ for the GAT + CFP combination and 8/11 $(72.7 \%)$ strains for the GAT + CFP-SUL combination. In contrast, the chequerboard titrations with these strains showed synergy for three strains $(27.3 \%)$ with the GAT + CFP combination and for six strains (54.5\%) with

Table 1. MICs of each agent alone

\begin{tabular}{|c|c|c|c|c|c|c|}
\hline \multirow[t]{3}{*}{ Group $(n)$} & \multicolumn{6}{|c|}{$\operatorname{MIC}\left(\mu \mathrm{g} \mathrm{ml}^{-1}\right)$} \\
\hline & \multicolumn{2}{|c|}{ GAT } & \multicolumn{2}{|c|}{ CFP } & \multicolumn{2}{|c|}{ CPF-SUL } \\
\hline & $\mathrm{MIC}_{50}$ & $\mathrm{MIC}_{90}$ & $\mathrm{MIC}_{50}$ & $\mathrm{MIC}_{90}$ & $\mathrm{MIC}_{50}$ & $\mathrm{MIC}_{90}$ \\
\hline $\begin{array}{l}\text { Susceptible } \\
(31)^{\star}\end{array}$ & 2 & 2 & 16 & 16 & 8 & 16 \\
\hline \multicolumn{7}{|l|}{ Resistant } \\
\hline GAT (6) & 8 & 16 & 16 & 16 & 16 & 16 \\
\hline CFP (2) & 2 & 2 & 64 & 64 & 16 & 16 \\
\hline $\begin{array}{l}\text { Miscellaneous } \\
(19) \dagger\end{array}$ & 8 & 8 & 64 & 128 & 32 & 32 \\
\hline
\end{tabular}

${ }^{\star}$ Susceptible to all agents tested.

$\dagger$ Resistant to all agents tested $(n=14)$ and resistant to GAT and CFP $(n=5)$.

Table 2. Results of chequerboard synergy testing

No antagonistic FIC indices $>4$ were found. FIC indices $\leqslant 0.5$ were considered synergistic, whilst those $>0.5-4$ were considered additive or indifferent.

\begin{tabular}{|c|c|c|c|c|}
\hline \multirow[t]{3}{*}{ Group $(n)$} & \multicolumn{4}{|c|}{ No. of strains with FIC indices of: } \\
\hline & \multicolumn{2}{|c|}{ GAT + CFP } & \multicolumn{2}{|c|}{ GAT + CFP-SUL } \\
\hline & $\leqslant 0.5$ & $>0.5-4$ & $\leqslant 0.5$ & $>0.5-4$ \\
\hline Susceptible (31) & 11 & 20 & 17 & 14 \\
\hline \multicolumn{5}{|l|}{ Resistant } \\
\hline GAT (6) & 2 & 4 & 4 & 2 \\
\hline CFP (2) & 2 & 0 & 2 & 0 \\
\hline Miscellaneous (19) & 6 & 13 & 11 & 8 \\
\hline
\end{tabular}


Table 3. Results of chequerboard and time-kill methodologies

Values in parentheses indicate the lowest concentration of each agent that yielded bactericidal activity at $24 \mathrm{~h}$ compared with that of the more active agent.

\begin{tabular}{|c|c|c|c|c|c|c|c|}
\hline \multirow[t]{3}{*}{ Strain } & \multicolumn{3}{|c|}{$\operatorname{MIC}\left(\mu \mathrm{g} \mathrm{ml}^{-1}\right)$} & \multicolumn{4}{|c|}{ Results for drug combination by two methods } \\
\hline & \multirow[t]{2}{*}{ GAT } & \multirow[t]{2}{*}{ CFP } & \multirow[t]{2}{*}{ CFP-SUL } & \multicolumn{2}{|c|}{$\mathrm{GAT}+\mathrm{CFP}$} & \multicolumn{2}{|c|}{ GAT + CFP-SUL } \\
\hline & & & & $\mathrm{C}$ & $\mathrm{T}$ & $\mathrm{C}$ & $\mathrm{T}$ \\
\hline \multicolumn{8}{|c|}{ Susceptible } \\
\hline 1 & 2 & 16 & 16 & Sy & Sy $(0.5 / 4)$ & Sy & Sy $(0.25 / 4)$ \\
\hline 2 & 2 & 16 & 8 & Ad & Sy $(0.5 / 4)$ & Ad & Ad \\
\hline 3 & 2 & 16 & 16 & Ad & Ad & Sy & Sy $(0.25 / 4)$ \\
\hline \multicolumn{8}{|c|}{ Resistant to GAT } \\
\hline 4 & 8 & 16 & 16 & Ad & Sy $(2 / 4)$ & Sy & $\operatorname{Sy}(1 / 4)$ \\
\hline 5 & 8 & 16 & 8 & Ad & Ad & Ad & Ad \\
\hline 6 & 8 & 16 & 16 & Ad & Ad & Ad & $\operatorname{Sy}(1 / 4)$ \\
\hline \multicolumn{8}{|c|}{ Resistant to CEF } \\
\hline 7 & 2 & 64 & 16 & Sy & Sy $(0.5 / 16)$ & Sy & Sy $(0.25 / 4)$ \\
\hline 8 & 2 & 64 & 32 & Sy & Sy $(0.5 / 16)$ & Sy & Sy $(0.25 / 4)$ \\
\hline \multicolumn{8}{|c|}{ Miscellaneous } \\
\hline 9 & 8 & 128 & 32 & Ad & Sy $(2 / 32)$ & Sy & Sy $(1 / 8)$ \\
\hline 10 & 8 & 64 & 16 & Ad & Ad & Ad & Sy $(2 / 4)$ \\
\hline 11 & 8 & 128 & 32 & Ad & Ad & Ad & Ad \\
\hline
\end{tabular}

Ad, Additive or indifferent; C, chequerboard titration; Sy, synergistic; T, time-kill studies.

the GAT + CFP-SUL combination. Concordance of the chequerboard and time-kill testing was demonstrated in 8/11 strains for the GAT + CFP combination and 9/11 strains for the GAT +CFP-SUL combination. Synergy between GAT and CFP-SUL was significantly $(P=0.011)$ more common than that between GAT and CFP.

\section{DISCUSSION}

Multidrug-resistant $P$. aeruginosa is an important causative agent of nosocomial infections. $\beta$-Lactams, aminoglycosides and fluoroquinolones have been the mainstay in the treatment of $P$. aeruginosa infections. Combination treatments are effective choices against nosocomial infections caused by multidrug-resistant isolates (PDR, 2004).

Combinations of fluoroquinolones with other agents have been investigated extensively (Dawis et al., 2003; Fish et al., 2002; Gradelski et al., 2001; Mayer \& Nagy, 1999). Most of the studies combining fluoroquinolones with aminoglycosides have shown indifference against members of the Enterobacteriaceae and against $P$. aeruginosa, whereas fluoroquinolones with anti-pseudomonal penicillins have been reported to be synergistic against 20-50\% of $P$. aeruginosa isolates (Neu \& Chin, 1994).

Gradelski et al. (2001) reported that the in vitro effect of GAT with CFP was synergistic against $40-60 \%$ of $P$. aeruginosa strains by time-kill methods. Furthermore, it was reported that amikacin in combination with GAT was synergistic against $P$. aeruginosa but the efficiency was least when compared with a GAT and $\beta$-lactam combination.

Two methods to detect in vitro synergy - a chequerboard and a time-kill assay - were performed for GAT + CFP and GAT + CFP-SUL combinations against 58 clinical isolates of $P$. aeruginosa. The combinations GAT $+\mathrm{CFP}$ and GAT + CFP-SUL were found to be synergistic for 36.2 and $58.6 \%$ of the isolates tested, respectively, using the chequerboard method. The rate of synergy of GAT + CFP correlates with earlier reports using this combination (Gradelski et al., 2001). There was a significant difference in synergy between the two combinations $(P=0.011)$. Our study showed that GAT at sub-MIC concentrations of $<0.25-0.5 \mu \mathrm{g} \mathrm{ml}{ }^{-1}$ was synergistic at $24 \mathrm{~h}$ when combined with CFP-SUL in 4/11 strains and had comparatively lower synergy rates when combined with CFP alone.

Synergy testing methods are not standardized for reproducibility and interpretation, and therefore it is extremely difficult to compare the results of these methods from different studies. The chequerboard test measures only the inhibitory concentration, and in time-kill studies the concentration is fixed and does not decrease as it would in vivo. The time parameter of $24 \mathrm{~h}$ can limit or alter the results of an experiment if regrowth occurs with one or both antimicrobial agents (Pankey \& Ashcraft, 2005). Although each of these methods uses different conditions and end points, there is frequent agreement between the results of the two methods. In our study, the agreement between these two methods was $72-81 \%$. 
In conclusion, our study demonstrated synergy in the combinations GAT + CFP and GAT + CFP-SUL against clinical isolates of $P$. aeruginosa. Although it is interesting that we could demonstrate in vitro synergy against some $P$. aeruginosa isolates, the mechanism of the exhibited synergy is unknown and needs to be explored. Also, no evidence of in vivo synergy has been found. Clinical studies are necessary to test the validity of these in vitro findings, as well as the significance of regrowth after $24 \mathrm{~h}$.

\section{REFERENCES}

Bajaksouzian, S., Visalli, M. A., Jacobs, M. R. \& Appelbaum, P. C. (1996). Antipneumococcal activities of cefpirome and cefotaxime, alone and in combination with vancomycin and teicoplanin, determined by checkerboard and time-kill methods. Antimicrob Agents Chemother 40, 1973-1976.

Dawis, M. A., Isenberg, H. D., France, K. A. \& Jenkins, S. G. (2003). In vitro activity of gatifloxacin alone and in combination with cefepime, meropenem, piperacillin and gentamicin against multidrug-resistant organisms. J Antimicrob Chemother 51, 1203-1211.

Eliopoulos, G. M. \& Moellering, R. C., Jr (1991). Laboratory methods used to assess the activity of antimicrobial combinations. In Antibiotics in Laboratory Medicine, 3rd edn, pp. 432-492. Edited by V. Lorian. Baltimore, MD: Williams \& Wilkins.

Fish, D. N., Choi, M. K. \& Jung, R. (2002). Synergic activity of cephalosporins plus fluoroquinolones against Pseudomonas aeruginosa with resistance to one or both drugs. J Antimicrob Chemother $\mathbf{5 0}$ 1045-1049.
Gradelski, E., Valera, L., Bonner, D. \& Fung-Tomc, J. (2001). Synergistic activities of gatifloxacin in combination with other antimicrobial agents against Pseudomonas aeruginosa and related species. Antimicrob Agents Chemother 45, 3220-3222.

Mayer, I. \& Nagy, E. (1999). Investigation of the synergic effects of aminoglycoside-fluoroquinolone and third-generation cephalosporin combinations against clinical isolates of Pseudomonas spp. J Antimicrob Chemother 43, 651-657.

CLSI (1999). Methods for Dilution Antimicrobial Susceptibility Tests for Bacteria that Grow Aerobically, 5th edn, approved standard M7-A5. Wayne, PA: Clinical and Laboratory Standards Institute.

CLSI (2000). Performance Standards for Antimicrobial Disk Susceptibility Test, approved standard M7-A5. Wayne, PA: Clinical and Laboratory Standards Institute.

Neu, H. C. \& Chin, N. X. (1994). In vitro activity of the new fluoroquinolone CP-99,219. Antimicrob Agents Chemother 38, 2615-2622.

Pankey, G. A. \& Ashcraft, D. S. (2005). In vitro synergy of ciprofloxacin and gatifloxacin against ciprofloxacin-resistant Pseudomonas aeruginosa. Antimicrob Agents Chemother 49, 2959-2964.

PDR (2004). Physicians' Desk Reference, 58th edn. Montvale, NJ: Thomsons.

Rossolini, G. M. \& Mantengoli, E. (2005). Treatment and control of severe infections caused by multiresistant Pseudomonas aeruginosa. Clin Microbiol Infect 11 (Suppl. 4), 17-32.

Visalli, M. A., Jacobs, M. R. \& Appelbaum, P. C. (1998). Determination of activities of levofloxacin, alone and combined with gentamicin, ceftazidime, cefpirome, and meropenem, against 124 strains of Pseudomonas aeruginosa by checkerboard and time-kill methodology. Antimicrob Agents Chemother 42, 953-955. 